\title{
Encuentros entre el sistema dominante de salud y otros sistemas de salud en el Perú (2000-2012)
}

\author{
Encounters between the dominant health system \\ and other health systems in Peru (2000-2012)
}

Tesania Velásquez ${ }^{1}$ /Guillermo Ríos ${ }^{2}$ /Natalia Incio Serra $^{3}$ / Max Renato Rivera Pantigozo ${ }^{4}$ / Diego Graña León ${ }^{5} /$ Sarah Moll León ${ }^{6}$

\section{Resumen}

En el Perú, la salud es entendida de diversas maneras y son muchas las prácticas que buscan preservarla. Si bien la oficialidad promueve un modelo biopsicosocial de la salud, gran parte de las comunidades comparten históricamente distintos significados y rituales con relación a la salud. El objetivo este artículo es explorar los encuentros y desencuentros entre el sistema oficial y otros sistemas de salud durante la primera década del siglo XXI. Para ello, se propone incorporar un enfoque comunitario que denuncia las características históricas y políticas que crean un vacío en el reconocimiento de otros

1 Dirección Académica de Responsabilidad Social (PUCP). Contacto: tvelazq@pucp.pe

2 Pontificia Universidad Católica del Perú. University College London. Contacto: rzguillermo@gmail.com

3 Candidata a magister en Educación por McGill University. Contacto: natserra@gmail.com

4 Licenciado en Ciencias Humanas con mención en Psicología Educacional por la PUCP. Contacto: maxriverapantigozo@gmail.com

5 Psicólogo clínico por la PUCP, magíster en educación intercultural por la universidad de Durham, Inglaterra. Contacto: diegrana@gmail.com

6 Licenciada en Psicólogía Clínica por la PUCP, magister en Psicología Comunitaria por la misma universidad. Contacto: sarah.moll@pucp.pe 
saberes. La discusión se centra en el contexto histórico del conflicto armado interno y el Informe final de la Comisión de la Verdad y Reconciliación (CVR).

Palabras clave: salud, sistemas alternativos de salud, conflicto armado interno, poder, Perú, siglo XXI, Comisión de la Verdad y Reconciliación (CVR)

\section{Abstract}

In Peru, health is understood in different ways and there are many practices that seek to maintain it. Each of these responds to spaces of identity and meaning shared by a community. While the official discourse promotes a bio-psychosocial model of health, many of the communities historically share different meanings and rituals in relation to health. The aim of this paper is to explore the encounters and misunderstandings between the official health system and other health systems during the first decade of $21^{\text {st. }}$ century. To this end, a community approach is proposed, which exposes the historical and political characteristics that deny the recognition of other knowledge. The discussion focuses on the internal armed conflict and the Final report of the Truth and Reconciliation Commission (CVR).

Key words: health, alternative health systems, political violence, power, Peru, internal armed conflict, Truth and Reconciliation Commission (CVR) 


\section{Introducción}

El Perú es un país pluricultural y multilingüe que se caracteriza por mantener una exclusión histórica de los grupos que no responden a la cultura hegemónica (Velázquez, Fernández, Mejía y Rivera, 2010). En este escenario de exclusión, hablar de salud nos remite a la pobreza y a la desigualdad como fenómeno social inmerso en la estructura y dinámica de la sociedad (Velázquez, 2007).

La salud como realidad sociocultural es afectada en su origen y desarrollo por la sociedad y la cultura. El modo como la sociedad encara colectivamente el mantenimiento de la salud y la respuesta a la enfermedad depende de intereses que se relacionan con lo político, lo económico y lo ideológico.

De manera general, la sociedad civil y el Estado interactúan para configurar, entre otros, el sector de la salud. El Estado, desde su monopolio de poder, negocia las demandas de los diversos grupos sociales para articular, eventualmente, respuestas a nivel burocrático y darle posterior atención a las demandas de la sociedad civil. Como señalan Perrone y Teixidó (2007), "no se trata de una estructura rígida, sino cambiante con los tiempos y los contextos históricos" (p. 1).

\section{Las diferentes producciones de sentido sobre la salud en el Perú}

La salud es entendida de diversas maneras y son muchas las prácticas que buscan preservarla. Cada una de ellas responde a espacios de identidad y de significado compartidos por una comunidad. Si bien la oficialidad promueve un modelo biopsicosocial de la salud, gran parte de las comunidades comparten históricamente distintos significados y rituales 
sobre la salud. Esta construcción de significados se basa en las relaciones de poder y dominación social, y devienen en sistemas de salud discrepantes (Parra y Pacheco, 2006).

Como consecuencia de esta discrepancia, otros sistemas de salud conforman "un mestizaje de conceptos, usos y costumbres" que abarcan diversas culturas y fenómenos migratorios históricos (Cabieses, 1993 en Arrieta, s. f.). Delgado (1991) afirma que otros sistemas de salud emplean recursos curativos basados en una cosmovisión mágico-religiosa, en la cual se insertan dichas prácticas. Debido a esto, el acercamiento entre el sistema de salud oficial y otros sistemas de salud es complejo, pues implica distintas formas de comprender el mundo (Golte, 2009).

En ese sentido, Mabit (1996) afirma que la subjetividad y la aproximación a través del sentimiento y la intuición han ido configurando la percepción de la dinámica de otros sistemas de salud. En contraste, este autor señala que una aproximación occidentalizada a la realidad implica un sesgo positivista y racional, que exagera el valor de las pruebas y concibe el tiempo de manera lineal. Estas diferencias epistemológicas han generado una escisión de los saberes vinculados a la salud.

El propósito de este estudio es aportar al estado de la investigación sobre los (des)encuentros entre el sistema oficial y los otros sistemas de salud en el Perú a partir de la salud mental comunitaria. En este sentido, se plantea la siguiente pregunta: ¿Cómo se ha construido la representación colectiva de la salud en el Perú durante el inicio del siglo XXI? 


\section{Conflicto armado interno y el Informe final de la Comi- sión de la Verdad y Reconciliación (CVR): Reconociendo otros sistemas de salud}

El conflicto armado interno azotó al Perú entre 1980 y 2000. Este fenómeno representa el evento de violencia más intenso y prolongado de nuestra historia (Comisión de la Verdad y Reconciliación, 2003). Las comunidades más afectadas por la violencia fueron la población andina quechua hablante. Estas poblaciones han estado históricamente expuestas a dinámicas de desigualdad, exclusión y discriminación. La ausencia del Estado y la indiferencia de gran parte de la población durante el conflicto ahondaron las fisuras sociales. Esta escisión abarcó distintos niveles y se manifestó también en el campo de la salud.

Desde un punto de vista funcional, el período del conflicto armado interno produjo un distanciamiento general entre muchas ciudades y provincias. Las poblaciones no viajaban por temor y consecuentemente un gran número de profesionales de la salud se alejó de la población de la sierra, cuyo contacto era percibido con recelo y desconfianza. Además de ello, otras formas de salud-enfermedad representaban lo desconocido, lo que durante ese período simbolizaba algo particularmente violento.

Durante el siglo XX, la mayoría de las iniciativas e intentos de diálogo e inclusión de los otros sistemas en el sistema oficial de salud se relaciona con individuos específicos. Por ese motivo, los acercamientos hacia otros modos de saludenfermedad no tuvieron un efecto significativo. Los estudios sobre otras formas de entender y practicar la salud parecieron desaparecer durante el período del conflicto armado interno. Con ello, la oficialidad ignoró todo aquello que no representara la concepción dominante de bienestar y salud. 
El contexto posconflicto armado y el reconocimiento de los efectos psicosociales en la población rural, andina y amazónica exigió el retorno de los profesionales de la salud y la salud mental a las regiones. Ante el reconocimiento de las necesidades de la población y la demanda de intervención en diferentes aspectos, se generó una nueva toma de consciencia que promovió la actuación de diversas instituciones y profesionales con otros contextos y poblaciones. Este encuentro con otros ámbitos de acción permitió el intercambio con otros saberes y formas de comprender la salud-enfermedad.

Las recomendaciones del Informe final de la Comisión de la Verdad y Reconciliación (IF-CVR) del 2003 señalan la importancia de las intervenciones psicosociales y comunitarias para hacer frente a las secuelas del conflicto. Además, plantea que las secuelas psicosociales no solo abarcan los niveles individuales y familiares, sino también el nivel comunal y social. Para comprender y atender adecuadamente la problemática de la salud mental en el contexto posconflicto resulta fundamental la incorporación de una mirada psicosocial, que trascienda el acercamiento y la atención individual. Solo a partir de un acercamiento complejo a la realidad se podrán conocer los efectos del conflicto en la población.

344 Los proyectos de intervención posconflicto armado interno tienen que incorporar un enfoque comunitario, que considere las relaciones históricas de la asimetría del poder. Este enfoque aborda la dimensión subjetiva y su influencia bidireccional en los procesos sociales (Grupo de Trabajo de Salud Mental de la Coordinadora Nacional de Derechos Humanos, 2006). 
Aldana (2001) menciona lo siguiente en relación con una intervención en una comunidad de personas desplazadas de zonas andinas rurales:

Propiciar una comunicación desde las categorías andinas permitió establecer muy paulatinamente la confianza de la población hacia el equipo de psicólogas [...]. La envidia era señalada como causa de la violencia, y el pago o ritual ofrecido a la pacha o madre tierra aparecía en el discurso de los pobladores como medio de curación. Al comprender la significación cultural de la envidia y el pago, el equipo de psicólogas buscó incorporar su forma de reparación en el proyecto (p. 108).

Esta autora resalta el valor de la espiritualidad en la recuperación de dicha comunidad desplazada y el efecto facilitador que tuvo en el diálogo con las psicólogas que participaron en la intervención comunitaria. Asimismo, señala que las secuelas del conflicto armado interno están presentes no solo en las comunidades afectadas, sino también en las poblaciones desplazadas, por lo que se propone incorporar la cosmovisión como una forma de restituir el vínculo social y darle significado a la vida. Resalta la importancia de la valoración y la inclusión de los códigos y costumbres propios en relación con la efectividad de una intervención comunitaria.

En esta línea, se hace referencia a tres iniciativas que tuvieron lugar en el contexto del posconflicto armado interno orientadas fundamentalmente a fortalecer capacidades locales y a promover la reconstrucción de los vínculos sociales. Para ello, los profesionales y trabajadores se vieron en la necesidad de reconocer y entender la cosmovisión, la cultura y, por ende, otros saberes en torno a las concepciones de la saludenfermedad. Esta acción, de acuerdo con el IF-CVR (2003), constituye la base de las políticas de reparación. 
Escribens, Portal, Ruiz y Velázquez (2008) exponen un proyecto sobre justicia y reparación en salud mental para mujeres víctimas de violencia sexual en una comunidad de la cuenca norte de Huancavelica. Los primeros hallazgos señalan que para los hombres y mujeres de la zona, el logro de la salud mental es el equilibrio entre los diferentes sentimientos de alegría, tristeza o pena (llaki), el susto o miedo (manchay) con la rabia o cólera (piñay). La salud mental se concibe como un balance que considera la enfermedad como parte de la vida. De esta forma, la salud implica la búsqueda de un equilibrio dinámico entre los diferentes elementos, ya que todos son importantes para el bienestar en la vida. La salud mental no se limita al ámbito individual, sino que se construye de forma colectiva y se asocia al reconocimiento positivo del otro y a los vínculos de confianza y respeto en la organización social.

En la misma línea, Pedersen (2006) apoya una propuesta ideográfica que se aparta de la generalización para abordar la problemática de forma profunda y compleja. Así, señala la importancia de revisar el uso de categorías diagnósticas que no tienen validez universal y propone una mirada distinta para aproximarse al dolor de las poblaciones culturalmente diferentes.

346 En un estudio sobre Ayacucho, el autor cuestiona la categoría de estrés postraumático para dar cuenta de la afectación por el conflicto armado interno y más bien encuentra que el llaki aparece relacionado con la experiencia de violencia política y con la condición de pobreza. Los llakis son pensamientos o recuerdos de tristeza o pena que se instalan como sentimientos en las personas. 
Considerando lo dicho anteriormente, la utilización de la palabra "susto" quizás sea una forma más fácil y directa para comunicarse con las poblaciones andinas afectadas. Escribens et al. (2008) plantean que el conflicto armado ha generado que todos en la comunidad estén "asustados" y desconfiados. De esta manera, se observa cómo el contacto con la comunidad y el intercambio de ideas puede generar un diálogo profundo entre los distintos saberes referidos a la salud. El reconocimiento y la valoración de sus conocimientos, experiencias y técnicas de tratamiento representan un vehículo de reparación.

Asimismo, Theidon (2004) plantea la importancia de no "medicalizar" los procesos de reconstrucción y reconciliación. Esta autora propone tratar de forma compleja los dolores y el malestar reportado por los pobladores y trascender la idea de calmar los síntomas físicos con el uso de medicamentos que abordan la problemática de manera superficial.

En este sentido, la mayor parte de las intervenciones reparadoras ocurre entre el paciente y su familia y amigos, lo que Kleinman (1980 citado en Theidon, 2004) llama el sector popular de los servicios de salud: los curanderos, las iglesias evangélicas, el trabajo conjunto, la reconstrucción de la vida ritual y las asambleas comunales.

Estas acciones e instituciones que surgen como iniciativas de la población para reconstruir el tejido social y crear una manera de vivir humanamente luego de los años de violencia representan escenarios que deben ser reconocidos y valorados por quienes trabajen con estas poblaciones, e incorporados de forma central en la intervención y en las políticas de reparación. 


\section{Discusión}

A pesar de los esfuerzos de incluir a los otros sistemas de salud que hemos mencionado, aún se reproduce la subordinación de las otras culturas respecto a la cultura oficial. Los encuentros y desencuentros siempre se dan en condiciones de desigualdad y las jerarquías entre ambas partes se mantienen. La hegemonía de la salud occidental no permite un diálogo con los otros sistemas de salud en las mismas condiciones. Esta lógica nos remite a un lugar común vinculado a la forma como se ha reconocido históricamente la diferencia y la diversidad en el Perú. Los otros sistemas de salud no son percibidos como negativos, ni se tiende a excluirlos deliberadamente. Por el contrario, existe una tendencia a su inclusión, pero bajo lo que hoy conocemos como inclusión adversa o inclusión inferiorizante.

Este fenómeno de exclusión parte de la idea del racismo sostenida por la lógica de la desigualdad, que acepta la presencia del otro mientras conserve su inferioridad. La inclusión inferiorizante reconoce al otro mediante la integración de este o de sus aspectos valiosos a la cultura dominante (Wieviorka, 1994).

La mirada desvalorizante y sesgada de los otros sistemas de salud se basa en un prejuicio generado por el desconocimiento y la discriminación. En este sentido, los otros sistemas de salud corresponden a los grupos sociales considerados inferiores y excluidos.

El Estado y el sistema de salud pública no tienen una aproximación integral que valide y reconozca a los otros sistemas de salud y a sus usuarios. Es decir, la oficialidad y el nivel institucional no propician la integración de otros saberes, sino que los mantienen en una posición de subordinación debido 
a la inclusión inferiorizante.

El reconocimiento de otros sistemas de salud es ante todo el reconocimiento de seres humanos distintos con sus respectivos valores, necesidades, problemas y cualidades. Eddowes y Saurín (2006) plantean que una intervención intercultural que considere las diferencias y ofrezca distintas alternativas en el ámbito de la salud sería también un reconocimiento a los derechos humanos de los pueblos tradicionales.

La inclusión social debe pasar necesariamente por el reconocimiento. Esto supone que la población encuentre sus valores y creencias reflejados en un sistema de atención que a la vez los reconozca. La inclusión no significa que la acción del Estado llegue a distintos puntos de la población con determinada cantidad de establecimientos de salud, sino que el poblador se sienta identificado con el servicio oficial de salud.

El encuentro entre la salud oficial y otros sistemas de salud depende, entre otros factores, del reconocimiento de las diferencias. Así, exige apertura y "un auténtico diálogo intercultural en el que ambas partes se respeten y se reconozcan sin prejuicio ni sojuzgamiento" (Zuluaga, 2009, p. 267). La integración implica un movimiento fluido que nutre y transforma continuamente ambas partes, y permite el surgimiento de una nueva forma que trasciende los diferentes sistemas de salud.

El reconocimiento de la diferencia en el encuentro con otros sistemas de salud nos invita a volver a preguntarnos: ¿Podemos nosotros hablar realmente por el subalterno?

Recibido: 05 de abril de 2018

Aprobado: 10 de agosto de 2018 


\section{Bibliografía}

Aldana, C.

(2001) Espiritualidad andina y reparación frente a la violencia política. En J. M. Mabit (Comp.), Ética, mal y transgresión. Memoria del Segundo Foro Interamericano Sobre Espiritualidad Indígena, 9 al 14 de noviembre de 1998. Tarapoto: CISEI/Takiwasi.

Cabieses, F.

(1993) Apuntes sobre Medicina Tradicional - La racionalización de lo irracional. Tomos I y II. Lima: Talleres de $A \& B$ S. A.

Comisión de la Verdad y Reconciliación (CVR)

(2003) Informe final de la Comisión de la Verdad y Reconciliación. Lima: CVR.

Delgado, H.

Medicina tradicional en Ayacucho: testimonio. Ayacucho: Universidad Nacional San Cristóbal de Huamanga.

Eddowes, J. \& Saurín, R. S.

(2006) Lo que sabemos nosotros es interminable: La medicina tradicional en territorio Shawi. Lima: Terra Nuova.

Escribens, P.; Portal, D.; Ruiz, S. y Velázquez, T.

(2008)

Reconociendo otros saberes. Salud mental comunitaria, justicia y reparación. Lima: Demus.

Golte, J.

(2009) ¿Es posible una articulación entre las medicinas "tradicionales" y las académicas? En R. Sánchez Garrafa y R. Sánchez Garrafa (eds.), Medicina tradicional andina. Planteamiento y aproximación (pp. 309-317). Cusco: Centro de Estudios Regionales Andinos Bartolomé de Las Casas (CBC), Centro de Medicina Andina (CMA). 
Grupo de Trabajo de Salud Mental de la Coordinadora Nacional de Derechos Humanos

(2006) Salud mental comunitaria en el Perú: aportes temáticos para el trabajo con poblaciones. Lima: Ministerio de Salud, Proyecto AMARES.

Mabit, J.

(1996) Investigación de las medicinas tradicionales, chamanismo y exploración del Mundo. Recuperado de http://www.takiwasi.com/docs/arti_esp/investigacion_mt_exploracion_mundo.pdf

Parra, L. y Pacheco, A.

(2006)

¿Monólogo o diálogo intercultural entre sistemas médicos? Un reto educativo para las ciencias de la salud. Revista Ciencias de la Salud 4(4): 110-121.

Pedersen, D.

(2006) Llaki-Nakary: explorando el mapa semántico de la aflicción y sufrimiento en la región alto andina de Ayacucho. Revista de Psiquiatría y Salud Mental Hospital Hermilio Valdizán, VII (1): 03-10.

Perrone, N. y Teixidó, L.

(2007) Estado y salud en la Argentina. Revista de la Maestría en Salud Pública, 10 (5): 1-46.

Sarrazín, J. P.

(2011) Transnacionalización de la espiritualidad indígena y turismo místico. Ponencia presentada en el IV Congreso de la Red Internacional de Migración y Desarrollo. "Crisis Global y Estrategias Migratorias: Hacia la Redefinición de las Políticas de Movilidad, Quito, Ecuador.

Spivak, G. (2003) ¿Puede hablar el subalterno? Revista Colombiana de Antropología 39: 297-364. 
Theidon, $\mathrm{K}$.

(2004) Entre prójimos. El conflicto armado interno y la politica de la reconciliación en el Perú. Lima: IEP.

Van der Hoogten, L. y Roersch, C.

(2009) La medicina andina y su futuro. En R. Sánchez Garrafa y R. Sánchez Garrafa (Eds.) Medicina tradicional andina. Planteamiento y aproximación (pp. 339-349). Cusco: Centro de Estudios Regionales Andinos Bartolomé de Las Casas (CBC), Centro de Medicina Andina (CMA).

Velázquez, $\mathrm{T}$.

(2007)

Salud mental en el Perú: dolor y propuesta. La experiencia de Huancavelica. Lima: CIES, CARE y PCS.

Velázquez, T.; Fernández, M. Z.; Mejía, G.; y Rivera, G.

(2010) Salud mental e interculturalidad. Ponencia presentada en el VII Congreso Internacional de la Red Latinoamericana de Antropología Jurídica RELAJU: Un Reto para Nuestras Sociedades: Identidades, Interculturalidad, Pluralismo Jurídico y Derechos Colectivos, Lima, Perú.

Wieviorka, M.

Racismo y exclusión. Estudios Sociológicos 12 (34).

Recuperado de http://codex.colmex.mx:8991/exlibris/aleph/a18_1/apache_media/YN8MHLQARHE842LGQFBMVAMC3E7QRU.pdf

352 Zuluaga, G.

(2009) Reflexiones para un diálogo entre los sistemas tradicionales de salud y la medicina occidental. En R. Sánchez Garrafa y R. Sánchez Garrafa (Eds.), Medicina Tradicional andina. Planteamiento y aproximación (pp. 255-271). Cusco: Centro de Estudios Regionales Andinos Bartolomé de Las Casas (CBC), Centro de Medicina Andina (CMA). 